\title{
Mugs and Plants: Object Semantic Knowledge Alters Perceptual Processing with Behavioral Ramifications
}

Dick Dubbelde ( $\boldsymbol{\sim}$ dubbelde@gwu.edu )

The George Washington University

\section{Sarah Shomstein}

George Washington University https://orcid.org/0000-0001-8278-6630

\section{Article}

Keywords: object semantic knowledge, neural processing, object recognition

Posted Date: March 19th, 2021

DOl: https://doi.org/10.21203/rs.3.rs-316736/v1

License: (c) (i) This work is licensed under a Creative Commons Attribution 4.0 International License.

Read Full License 
Title:

2

3

4
Mugs and Plants: Object Semantic Knowledge Alters Perceptual Processing with Behavioral Ramifications

Dick Dubbelde and Sarah Shomstein

Department of Psychological and Brain Sciences

The George Washington University

\section{Corresponding author:}

\section{Dick Dubbelde}

Department of Psychology

The George Washington University 2125 G St. NW

Washington, DC 20052

E-mail: dubbelde@gwu.edu 


\section{Abstract}

22 Neural processing of objects with action associations is thought to recruit dorsal visual regions

23 more than objects without such associations. We hypothesized that because the dorsal and

24 ventral visual pathways have differing proportions of magno- and parvo-cellular input, there

25 should be behavioral differences in perceptual tasks between manipulable and non-manipulable

26 objects. This hypothesis was tested using gap detection task, suited to the spatial resolution of the

27 ventral parvocellular processing, and object flicker discrimination task, suited to the temporal

28 resolution of the dorsal magnocellular processing. Directly predicted from the cellular

29 composition of each pathway, a non-manipulable object advantage was observed in tasks relying

30 on spatial resolution, and a manipulable object advantage in temporal discrimination. We also

31 show that these relative advantages are modulated by either reducing object recognition through

32 inversion or by suppressing magnocellular processing using red light. These results establish

33 perceptual differences between objects dependent on prior knowledge and experience. 
Object perception and recognition underlies effective engagement with our complex

environment. When looking at a potted plant, for example, precise access to long term semantic

memory may be needed to facilitate a more detailed identification of the plant to know how often

39 it needs to be watered. However, when reaching for a mug to have your morning coffee, detailed identification may be less important (e.g., which one of your 10 favorite mugs it is), but what is

41 more pertinent is computing a motor plan to bring the mug to your mouth without spilling. The visual object processing necessary to identify plants and to drink from mugs is understood to

43 progress along two distinct but interacting neural processing pathways, each of which is understood to subserve different end goals (Mishkin \& Ungerleider, 1982; Kravitz et al., 2011). While the hypothesis of biased processing across the two pathways is widely accepted, it is unclear whether there are behavioral ramifications that are directly related to object processing

47 being biased to different pathways. Here, we provide evidence that object semantic knowledge evokes differences in object perception based on the recruited regions across the dorsal or ventral streams. Importantly, we gain insight into neural processing by using purely behavioral methods that are custom tailored to the response properties of the neurons in both the dorsal (higher temporal resolution) and ventral pathways (higher spatial resolution). feature selectivity and projects anteriorly from the occipital visual areas toward the hippocampus and the inferior temporal cortex (Kravitz et al., 2013). Damage to cortical regions along the ventral pathway results in visual agnosia, evidenced by difficulties in object recognition but preserved motor interactions with objects (Goodale \& Milner, 1992; 1994; Karnath et al., 2009).

57 Patients with ventral pathway damage but an intact dorsal pathway continue to manipulate, 
greatly impaired (James et al., 2003). The dorsal pathway, in turn, colloquially known as the

60 'where' or 'how' pathway, courses from the occipital visual areas into the parietal cortex. The

61 dorsal pathway is commonly understood to subserve object processing for the purposes of

62 reaching, grasping, and manipulation. While both pathways contribute to visual perception, the

63 demands of perceiving a specific object can differentially engage the two pathways such that the

64 dorsal pathway provides greater contribution for visual processing of objects that are directly

65 relevant for object manipulation (e.g., a hammer, a plunger, or a mug). Evidence for dynamic

66 recruitment of processing from one pathway more than the other has been garnered from a wide

67 range of techniques and paradigms. For example, while presenting participants with objects of

68 various categories, including tools, places, animals, and faces, an increase in dorsal pathway

69 processing, specifically in the left ventral premotor and left posterior parietal cortices, has been

70 observed exclusively in response to tool presentation (Chao \& Martin, 2000). Similar results

71 which evidence a dorsal bias for manipulable objects have been found in functional magnetic

72 resonance imaging studies (Noppeney et al., 2006a; Mahon et al., 2007, Almeida et al., 2013,

73 Chen et al., 2018) and with various other paradigms including interocular suppression (Fang and

74 He, 2005) and continuous flash suppression (Almeida et al., 2008; Almeida et al., 2010).

75 While neuroimaging and neurophysiological evidence has been convincing in showing

76 selectivity for objects in various areas across the two streams, the impact that differential

77 processing across the two pathways has on object perception and subsequent behavior has not

78 been characterized. Hypotheses regarding how dynamic recruitment of the dorsal and ventral

79 pathways differentially influences perception and behavior are based on the anatomy and

80 physiology of the two visual pathways. Considering cellular physiology, there is an asymmetry

81 in innervation of the two pathways that overlaps with the separate magnocellular and 
82 parvocellular processing pathways identified in anatomical architecture of visual processing

83 (Maunsell et al., 1990; Baizer et al., 1991; Ferrera et al., 1992). The asymmetry in cellular

84 composition across the two pathways endows each pathway with different response properties in

85 accordance with cell stimulus preferences within the magno- and parvo-cellular pathways. The

86 magno- and parvo-cellular pathways originate from different types of ganglion cells within the

87 retina and course separately through different layers of the lateral geniculate nucleus (Leventhal

88 et al., 1981; Perry et al., 1984), innervating separate layers of V1 (Blasdel \& Lund, 1983). From

$89 \mathrm{~V} 1$, the parvocellular pathway can be followed into area V2, through area V4, and then into the

90 inferior parietal cortex while the magnocellular pathway can be traced into different regions of

91 area V2, through areas V3d and MT, and then into the posterior parietal cortices (DeYoe \& van

92 Essen, 1988; Livingstone \& Hubel, 1988).

93 Differentiation in cellular innervation of the two processing pathways leads to different

94 response properties that convey information of different spatial and temporal resolutions. The

95 magnocellular channel is derived from the parasol ganglion cells with relatively large receptive

96 fields, spanning large regions of the retina (Nassi \& Callaway, 2009). The cells of the

97 magnocellular channel are more myelinated, and have less neuronal convergence than the

98 parvocellular channel (Maunsell et al., 1999a). The features of the parasol retinal ganglion cells

99 enable the dorsally biased magnocellular pathway to encode information with a higher temporal

100 resolution than that of the parvocellular channel (Pokorny and Smith, 1997; but see Maunsell et

101 al., 1999b). Conversely, the parvocellular channel is derived from the midget retinal ganglion

102 cells, which receive input primarily from cone receptors and have smaller receptive fields (Nassi

$103 \&$ Callaway, 2009). The features of the midget retinal ganglion cells enable the ventrally biased

104 parvocellular channel to encode information with a higher spatial resolution than that of the 
105 magnocellular channel (Derrington \& Lennie, 1984; Leonova et al., 2003; McAnany \&

106 Alexander, 2008).

107 Due to the asymmetric contributions of the magno- and parvo-cellular pathways to the

108 dorsal and ventral pathway processing pathways, we hypothesize that semantic knowledge of

109 object utility (manipulable or non-manipulable) determines processing bias to either dorsal or

110 ventral pathway processing and thus has consequences for perceptual processing. Thus, if

111 semantic knowledge of an object's utility determines to which of the two pathways the bias will

112 accrue, then: (1) manipulable objects, that elicit a higher degree of magnocellularly biased dorsal

113 processing, should be processed with higher temporal resolution; and (2) non-manipulable

114 objects, that rely more on parvocellularly biased ventral processing, should be processed with

115 higher spatial resolution. Across five experiments, this hypothesis is tested by comparing

116 processing of temporal and spatial resolution in two object groups: manipulable and non-

117 manipulable objects. Each group consisted of ten line-drawings of objects which were carefully

118 controlled for low-level perceptual differences (width of the gap line, luminance, size, and aspect

119 ratio which a measure of elongation; see Methods for details). Due to the fact that our hypotheses

120 are predicated on the idea that action associations bias processing across the two streams, the

121 objects were chosen based on how often they are manipulated with the hands when they are

122 encountered rather than by their association to any particular semantic grouping. The

123 manipulable object set included objects that are typically manipulated by the hands when they

124 are encountered, such as a hammer, a plunger, and a mug (Fig. 1A). The non-manipulable object

125 set included objects that are rarely manipulated with the hands when they are encountered, such

126 as a fire hydrant, a picture frame, and a potted plant (Fig. 1A). 


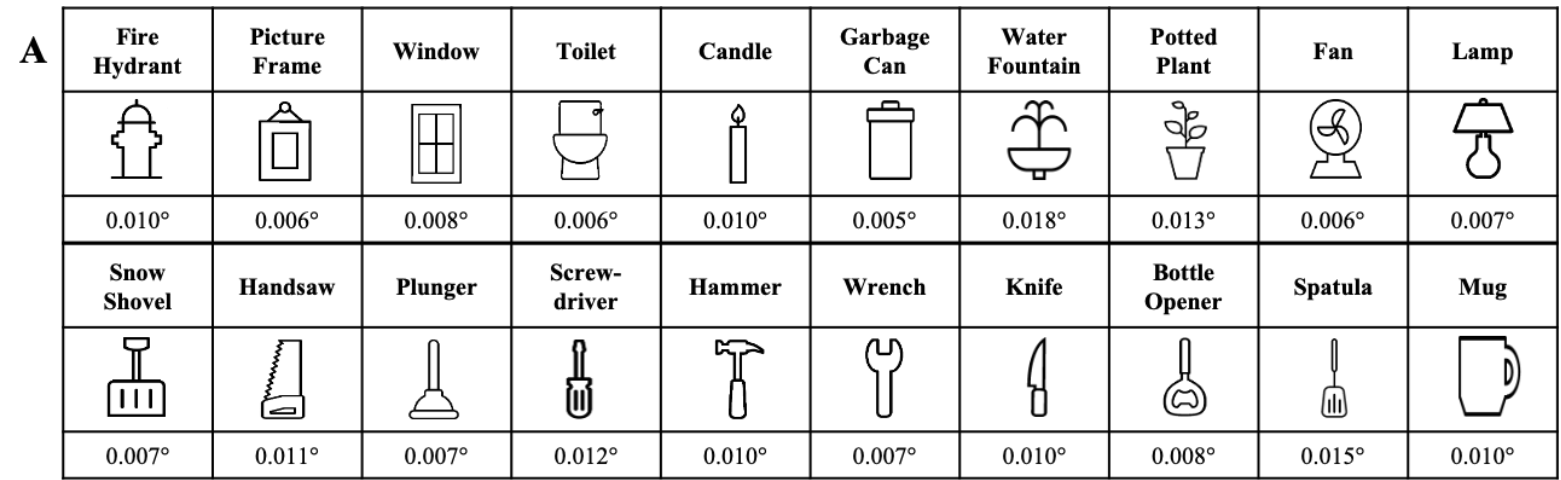

B

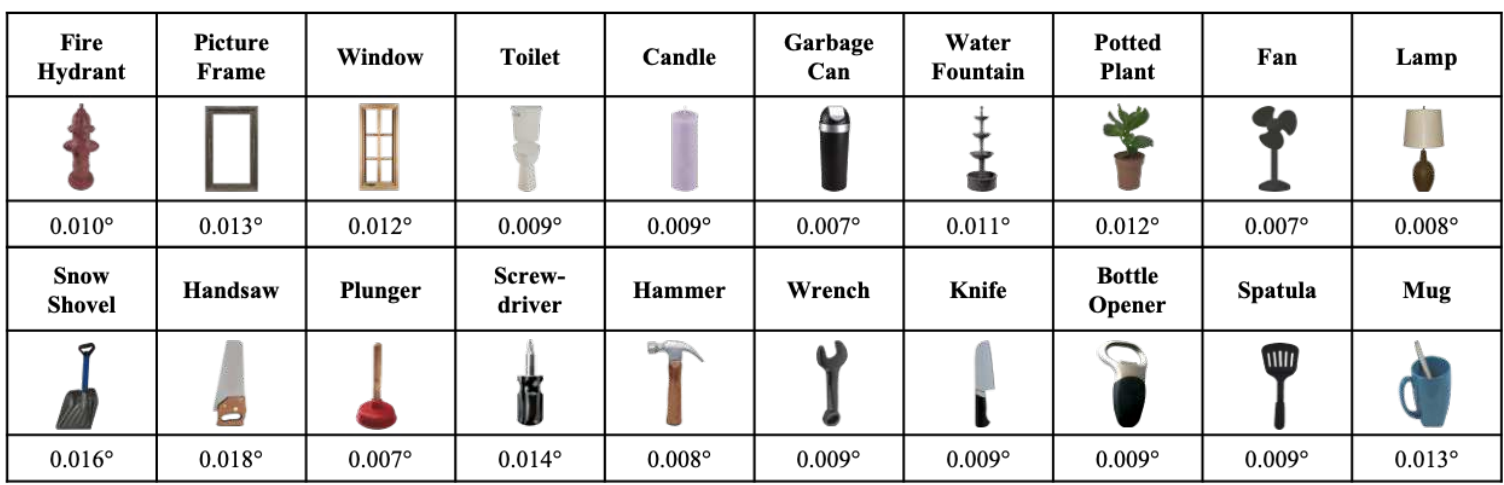

Figure 1 Stimulus set used in the experimental paradigms. (A) Line drawings of manipulable and non-manipulable objects used in Exp. 1. (B) Pictures of real-world manipulable and non-manipulable objects used in the second control manipulation for Exp. 1. The numbers beneath each set of objects correspond to the size of the spatial gap titrated to each object.

Experiment 1 employed two paradigms, each designed to take advantage of the unique

132 stimulus response properties of the two visual processing pathways and their corresponding

133 cellular composition. To measure ventral pathway engagement a gap detection task was

134 employed. Each object appeared to the left or the right of fixation, and would either have an

135 uninterrupted bottom contour or have a small spatial gap in the center of the bottom line of the object (Fig. 2A). Object group was counterbalanced such that manipulable objects were seen as

137 commonly as non-manipulable objects, and objects within each group were counterbalanced

138 such that all objects were seen an equal number of times. The size of the gap was titrated, in a

139 separate procedure for each object individually in an independent group of participants, to a

140 specific threshold making the task difficult but not impossible (See Methods for details).

141 Participants' task was to report the presence or absence of the gap. If object semantic knowledge 
142 determines the route of processing, then the presence of a spatial gap on non-manipulable objects

143 will be detected with higher sensitivity as compared to manipulable objects because processing

144 will be biased to ventral pathway's parvocellular processing.

145 To measure dorsal pathway engagement, a blink detection task was used. Similar to the

146 spatial gap detection task, participants detected the presence of a short blink. During a blink trial,

147 the object was presented for $80 \mathrm{~ms}$, then removed for the short duration of $16 \mathrm{~ms}$, then

148 redisplayed for additional $48 \mathrm{~ms}$. The duration of the blink was titrated with a staircase

149 procedure, with the shortest titrations limited by the lower bound of the monitor's refresh rate.

150 Thus, shortest blink duration was set to $16 \mathrm{~ms}$. During a non-blink trial, the objects remained on

151 the screen for the total duration of $144 \mathrm{~ms}$. Similar to the spatial gap paradigm, participants

152 responded with a button press to indicate whether a blink was detected. If object semantic

153 knowledge determines the route of processing, then the presence of a temporal gap during the

154 presentation of manipulable objects will be detected with higher sensitivity as compared to non-

155 manipulable objects because processing will be biased to magnocellularly-dominated dorsal

156 pathway. For both tasks, participants were given feedback on incorrect trials. Each participant

157 performed both tasks ( 3 blocks each), in a counterbalanced order, across six blocks. 
A

B
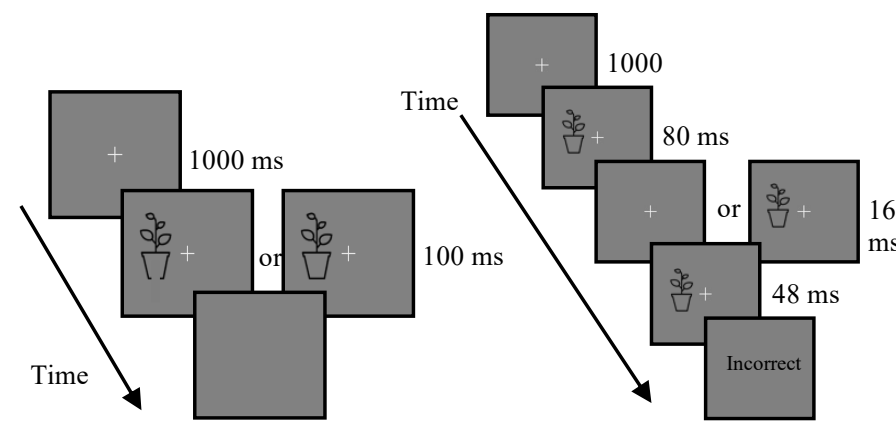

C

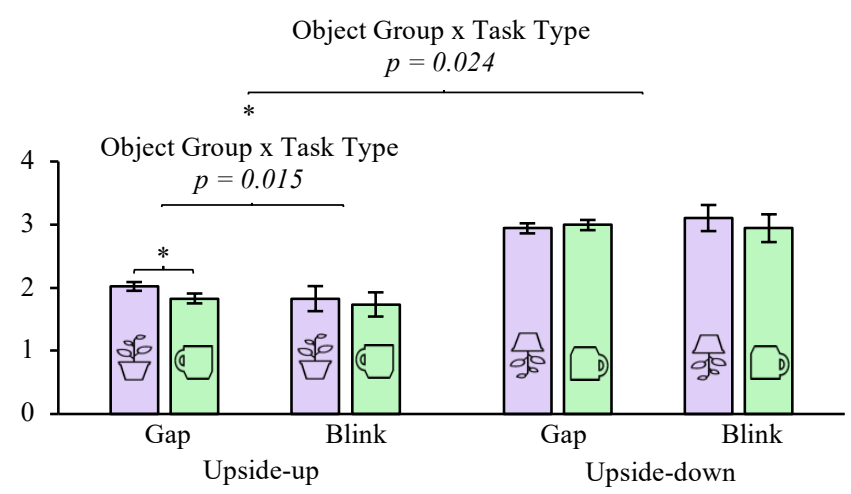

Figure 2. The paradigm and results for Experiments 1 and 2. Participants maintained fixation on the center cross. An object appeared to the left or right of fixation. A) For the spatial-gap paradigm, participants indicated by keypress if the bottom line of the presented object contained a gap. B) for the temporal paradigm, participants were to indicate whether the object flickered. C) Results for Experiment 1 $(n=26)$ and $2(n=30)$. For Experiment $1(C$, left $)$, there was a significant main effect of task type $(F(1,25)=50.953, p<$ $\left.0.001, \eta_{p}^{2}=0.671\right)$ such that d' sensitivity was higher for blinks than for gaps $\left(M_{\text {gaps }}=1.93 ; M_{\text {blinks }}=2.97\right)$; and a significant two-way interaction between object group and task type $\left(F(1,25)=6.772, p=0.015, \eta_{p}^{2}=0.213\right)$, driven by the difference between object groups in the gap condition $\left(F(1,25)=9.888, p=0.004, \eta_{p}^{2}=0.283\right.$, with nonmanipulable objects having a higher d' for gaps than manipulable objects $\left(M_{\text {non-manipulable }}=2.021 ; M_{\text {manipulable }}=\right.$ 1.831). For Experiment 2 (C, right), there was a significant main effect of object group $\left(F(1,25)=5.860, p=0.023, \eta_{p}^{2}\right.$ $=0.190)$ such that d' was higher for non-manipulable objects than for manipulable objects $\left(M_{\text {non-manipulable }}=2.466\right.$; $\left.M_{\text {manipulable }}=2.340\right)$ (Fig. $2 \mathrm{C}$, right). There was also a significant main effect of task type $(F(1,25)=82.298, p<$ $\left.0.001, \eta_{p}^{2}=0.767\right)$ such that d' was higher for blinks than for gaps $\left(M_{\text {gaps }}=1.782 ; M_{\text {blinks }}=3.024\right)$. There was no significant interaction between object group and task type $\left(F(1,25)=0.508, p=0.483, \eta_{p}^{2}=0.020\right)$. Additionally, across the two experiments, with orientation as a between subjects factor, there was a significant three-way interaction between object group, task type, and orientation $(F(1,50)=$ 5.381, $\left.p=0.024, \eta_{p}^{2}=0.097\right)$ such that orientation significantly reduced the effect for the inverted objects.

The spatial and temporal paradigms used d' as a measure of perceptual sensitivity (Fig.

160 2). A two-way repeated-measures analysis of variance (ANOVA) was conducted on d' with

161 object group (manipulable, non-manipulable) and stimulus type (gap, blink) as within-subject 
167 between object group and task type $\left(F(1,25)=6.772, p=0.015, \eta_{p}{ }^{2}=0.213\right)$ was observed, 168 driven by the difference between object groups in the gap condition $(F(1,25)=9.888, p=0.004$, $169 \eta_{p}^{2}=0.283$ ), with non-manipulable objects having a higher d' for gaps than manipulable objects

$170 \quad\left(\mathrm{M}_{\text {non-manipulable }}=2.021 ; \mathrm{M}_{\text {manipulable }}=1.831\right)$. The interaction effect and the driving main effect are 171 consistent with the prediction that non-manipulable objects, given reliance on ventral pathway 172 processing, should yield higher sensitivity in the detection of spatial gaps than manipulable 173 objects. Notably, the expected higher sensitivity to temporal gaps in the manipulable object set 174 was not supported. Given the high sensitivity for the temporal task type (d' $=2.941$ for non175 manipulable objects and d' $=2.993$ for manipulable objects), the lack of difference between the 176 object groups in the blink condition was likely due to the temporal task being overly easy (i.e., 177 ceiling effect; see Exp. 4 for a manipulation that avoids ceiling effects).

178 While Experiment 1 provides evidence for biased engagement of the ventral pathway for 179 non-manipulable object perception during a temporal task, it could be argued that despite careful 180 low-level feature controls (e.g., luminance, size, elongation) an uncontrolled low-level difference 181 between manipulable and non-manipulable objects is responsible for driving the manipulable vs. 182 non-manipulable advantage in the spatial gap task. In order to rule out this possibility and, 183 simultaneously, to further probe the hypothesis that semantic knowledge of object utility 184 (manipulable or non-manipulable) biases the pathway that will ultimately process the object, we 185 conducted two control experiments. In addition to serving as a low-level control and a further 186 test of the hypothesis, these experiments provide internal replications of the reported finding (see 187 Open Science Collaboration, 2015). In the first control manipulation, the same paradigm and objects from Experiment 1 were 189 used with the manipulation of inverting objects (upside-down). The inversion preserved the low- 
190 level features of each object while impairing participants' ability to rapidly recognize and access

191 their semantic knowledge of the objects' function. Inversion has been found to interfere with

192 recognition of faces and objects (Diamond and Carey, 1986). Following from the hypothesis that

193 semantic knowledge of object's utility drives the perceptual difference between manipulable and

194 non-manipulable objects, we predict that object inversion should reduce the difference between

195 manipulable and non-manipulable objects. A two-way repeated-measures ANOVA was

196 conducted on d' with object group (manipulable, non-manipulable) and task type (gap, blink) as

197 within-subject variables. The ANOVA revealed a significant main effect of object group $(F(1$,

$19825)=5.860, p=0.023, \eta_{p}{ }^{2}=0.190$ ) such that d' was higher for non-manipulable objects than for

199 manipulable objects $\left(\mathrm{M}_{\text {non-manipulable }}=2.466 ; \mathrm{M}_{\text {manipulable }}=2.340\right)($ Fig. $2 \mathrm{C}$, right $)$. There was also

200 a significant main effect of task type $\left(F(1,25)=82.298, p<0.001, \eta_{p}{ }^{2}=0.767\right)$ such that d' was

201 higher for blinks than for gaps $\left(\mathrm{M}_{\mathrm{gaps}}=1.782 ; \mathrm{M}_{\mathrm{blinks}}=3.024\right)$. As predicted, there was no

202 significant interaction between object group and task type $\left(F(1,25)=0.508, p=0.483, \eta_{p}{ }^{2}=\right.$

203 0.020). In order to statistically demonstrate that results of the inversion experiment are indeed

204 different from those observed in Experiment 1, data were subjected to a between-subject

205 ANOVA with object orientation (upright, inverted) as a between subjects variable and object

206 group and task type as within subject factors. The ANOVA revealed a significant three-way

207 interaction between object group, task type, and orientation $\left(F(1,50)=5.381, p=0.024, \eta_{p}{ }^{2}=\right.$

208 0.097) such that, as predicted, orientation significantly reduced the effect for the inverted objects.

209 In the second control manipulation, line drawings were replaced by real-world images of

210 corresponding objects, such that a line drawing of a candle was replaced with a picture of a

211 candle, etc. (Fig. 1B). The prediction remained the same as in the original experiment, if object

212 semantic knowledge determines which visual processing pathway object processing is biased to 
213 then non-manipulable objects will be biased to the ventral pathway leading to higher sensitivity

214 (as measured by d') in the spatial gap task, replicating the pattern of performance observed in

215 Experiment 1. A two-way repeated-measures ANOVA was conducted on d' with object group

216 (manipulable, non-manipulable) and task type (gap, blink) as within-subject variables. The

217 ANOVA revealed a significant main effect of object group $\left(F(1,29)=28.211, p<0.001, \eta_{p}{ }^{2}=\right.$

218 0.493) with non-manipulable objects having a higher sensitivity than manipulable objects $\left(\mathrm{M}_{\text {non- }}\right.$

219 manipulable $\left.=2.361, \mathrm{M}_{\text {manipulable }}=2.046\right)($ Fig. 3). There was also a significant main effect of task

220 type $\left(F(1,29)=8.413, p=0.007, \eta_{p}{ }^{2}=0.225\right)$ with blinks having a higher average sensitivity

221 than gaps $\left(\mathrm{M}_{\mathrm{blinks}}=2.409, \mathrm{M}_{\mathrm{gaps}}=1.998\right)$. Importantly, a two-way interaction between object

222 group and task type was also significant $\left(F(1,29)=13.061, p=0.001, \eta_{p}{ }^{2}=0.311\right)$, driven by a

223 simple main effect in the gap condition $\left(F(1,29)=46.424, p<0.001, \eta_{p}{ }^{2}=0.616\right)$ such that non-

224 manipulable objects had a higher d' for gaps than did the manipulable objects $\left(\mathrm{M}_{\text {non-manipulable }}=\right.$

$2252.238 ; M_{\text {manipulable }}=1.758$ ). In order to statistically demonstrate that results observed using real

226 objects are indistinguishable from the results observed using the line-drawings in Experiment 1,

227 the data were subjected to a between-subject ANOVA with stimulus type (line drawings, real

228 object images) as a between subjects variable and object group and task type as within subject

229 factors. The ANOVA revealed no significant three-way interaction between object group, task

230 type, and stimulus type $\left(F(1,54)=0.454, p=0.503, \eta_{p}{ }^{2}=0.000159\right)$. These results replicate

231 Experiment 1, providing strong additional support for the hypothesis that the perceptual

232 differences between manipulable objects and non-manipulable objects is due to the semantic

233 content of the objects rather than low-level features. 
Figure 3. Results from the second control manipulation of experiment one in which real object images were used instead of line drawing images. There was a significant main effect of object group $\left(F(1,29)=28.211, p<0.001, \eta_{p}^{2}=0.493\right)$ with

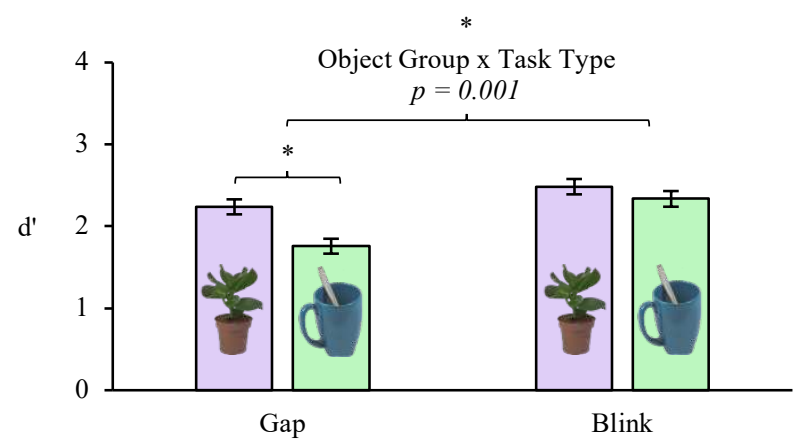

non-manipulable objects having a higher sensitivity than manipulable objects ( $\left.M_{\text {non-manipulable }}=2.361, M_{\text {manipulable }}=2.046\right)$ (Fig. 3). There was also a significant main effect of task type ( $F(1$, 29) $\left.=8.413, p=0.007, \eta_{p}{ }^{2}=0.225\right)$ with blinks having a higher average than gaps $\left(M_{\text {blinks }}=2.409, M_{\text {gaps }}=1.998\right)$. The two-way interaction between object group and task type was also significant $\left(F(1,29)=13.061, p=0.001, \eta_{p}^{2}=0.311\right)$, driven by a simple main effect in the gap condition $(F(1,29)=46.424, p<$ $\left.0.001, \eta_{p}^{2}=0.616\right)$ such that non-manipulable objects had a higher d' for gaps than did the manipulable objects $\left(M_{n o t}\right.$ $=2.238 ; M_{\text {manipulable }}=1.758$ ). While the first three experiments yielded strong supporting evidence that gap detection is

236 better on non-manipulable objects than manipulable objects, perhaps resulting from bias toward

237 the ventral pathway, manipulable objects failed to elicit an advantage in temporal resolution,

238 failing to provide evidence of a dorsal pathway bias for manipulable objects. One explanation for

239 the null effect is that the temporal gap detection task was too easy (ceiling effect), evidenced by

240 high d' values in the temporal task. To increase task difficulty, and move away from the ceiling

241 performance, the blink paradigm was re-designed to a discrimination task ${ }^{1}$. Objects were

242 presented for $80 \mathrm{~ms}$, removed from the screen for either a $16 \mathrm{~ms}$ or $48 \mathrm{~ms}$ blink, and redisplayed

243 for $48 \mathrm{~ms}$ (Fig. 4). Participants' task was to indicate whether the blink duration was short or long.

244 The prediction was that manipulable objects should have a performance benefit over non-

245 manipulable objects due to the increased magnocellular input, and therefore temporal resolution,

246 of the dorsal pathway. Due to the difficulty of the task (mean accuracy $77 \%$ ), evidenced by low

247 d' values, analyses were conducted on RT. A two-way repeated-measures ANOVA was

\footnotetext{
${ }^{1}$ We thank Ed Awh, from the University of Chicago, for the helpful suggestion.
} 
249 long) as within-subject variables. Only the RTs for correct responses were analyzed.

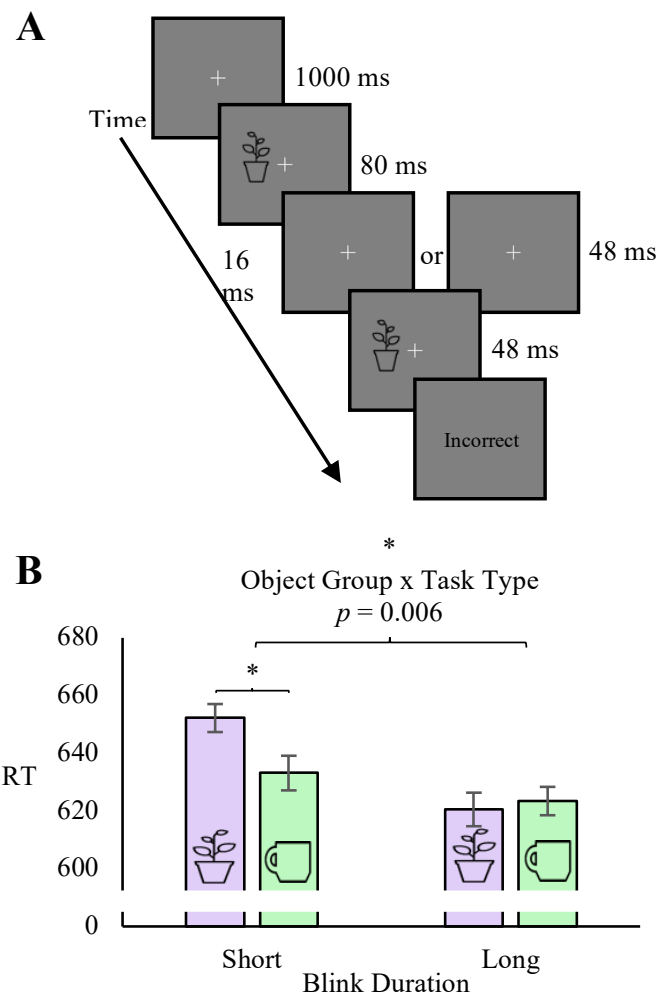

Figure. 4. The paradigm and results for experiment 4. A) Participants maintained fixation on the center cross. An object would then appear to the left or right of fixation. The objects would flicker by being removed from the screen for $16 \mathrm{~ms}$ or $48 \mathrm{~ms}$. Participants were to indicate by keypress if the object flicker duration was short or long. B) There was no significant main effect of object group $(F(1,24)=$ 4.080, $\left.p=0.055, \eta_{p}{ }^{2}=0.145\right)$. There was $a$ significant main effect of blink duration $\left(F(1,24)=4.850 p=0.038 \eta_{p}^{2}=0.168\right)$ such that the short duration elicited a higher RTs than did the long duration $\left(M_{\text {short }}=642.68 \mathrm{~ms} ; M_{\text {long }}=621.997 \mathrm{~ms}\right)$. The two-way interaction between object group and blink duration was significant $\left(F(1,24)=9.011, p=0.006, \eta_{p}^{2}=0.273\right)$ driven by a simple main effect in the short blink condition $(F(1,24)=11.710, p=$ $\left.0.002, \eta_{p}^{2}=0.328\right)$ such that manipulable objects had a lower RT for short blinks than did the non-manipulable objects $\left(M_{\text {manipulables }}=633.150 ; M_{\text {non-manipulable }}=\right.$ 652.210).

The ANOVA revealed no significant main effect of object group $(F(1,24)=4.080, p=$ duration $\left(\mathrm{M}_{\text {short }}=642.68 \mathrm{~ms} ; \mathrm{M}_{\text {long }}=621.997 \mathrm{~ms}\right)$. The two-way interaction between object group and blink duration was significant $\left(F(1,24)=9.011, p=0.006, \eta_{p}{ }^{2}=0.273\right)$ driven by a

256 simple main effect in the short blink condition $\left(F(1,24)=11.710, p=0.002, \eta_{p}{ }^{2}=0.328\right)$ such

257 that manipulable objects had a lower RT for short blinks than did the non-manipulable objects

$258\left(\mathrm{M}_{\text {manipulable }}=633.150 ; \mathrm{M}_{\text {non-manipulable }}=652.210\right)$. In accuracy, no effect was observed for object 
$260=0.059)$, nor the interaction $\left(F(1,24)=2.000, p=0.170, \eta_{p}{ }^{2}=0.077\right)$, suggesting that all

261 significant effects were absorbed by the response time measure and no speed-accuracy tradeoffs

262 were observed, indicating consistency with response time results. These results, specifically the

263 simple main effect in the short gap condition with manipulable objects having a lower RT than

264 non-manipulable objects, supports our hypothesis that manipulable object processing would

265 elicit higher temporal resolution than non-manipulable object processing. The lack of a

266 difference in the long duration condition is likely due to a ceiling effect, similar to the lack of

267 temporal effect in Experiment 1.

268 The last test of our hypotheses is derived from the neurophysiological differences

269 between the dorsal and ventral pathways. It was reasoned that if the differences in spatial gap

270 sensitivity for non-manipulable objects are mechanistically derived from the magnocellular and

271 parvocellular dichotomy of the two visual processing pathways, then the effect should be

272 modulated through manipulation of processing within the pathways. Ambient red light has been

273 shown to suppress activity in the magnocellular processing channel because of the large number

274 of visually responsive cells with red inhibitory surrounds in their receptive fields (Wiesel \&

275 Hubel, 1966). Therefore, presenting objects with a red background should suppress the

276 contribution from the parietal magnocellular pathway, whereas a background of any other color

277 should have no impact on processing. To test this prediction, the spatial resolution paradigm

278 from Experiment 1 was used with the manipulation of background color varying between green

279 or red (Fig. 5A). Due to the suppression of the magnocellular channel by red light, it was

280 predicted that the color of the background should modulate the perceptual difference in spatial

281 gap detected observed in Experiment 1. Namely, suppressing the contribution of the dorsal

282 pathway should exacerbate the difference between manipulable and non-manipulable objects. A 
two-way repeated-measures ANOVA was conducted on d' with object group (manipulable, non-

revealed a significant main effect of object group $\left(F(1,25)=17.171, p<0.001, \eta_{p}{ }^{2}=0.407\right)$

2.25, $\mathrm{M}_{\text {manipulable }}=2.02$ ) (Fig. 5B). There was no significant main effect of background color

$\left(F(1,25)=2.138, p=0.156, \eta_{p}{ }^{2}=0.079\right)$ but, as predicted, there was a significant two-way

interaction between object group and background color $\left(F(1,25)=4.444, p=0.045, \eta_{p}{ }^{2}=0.151\right)$

such that the effect was increased with the red background. It was hypothesized that if the

perceptual differences between manipulable and non-manipulable objects are mechanistically

292 derived from the magno- and parvo-cellular processing in the two visual pathways, then

293 suppression of the magnocellular processing with red light should modulate the effect. The

294 results of Experiment 5 support our hypothesis by demonstrating an increase of the perceptual

295 difference between manipulable and non-manipulable objects with red light.

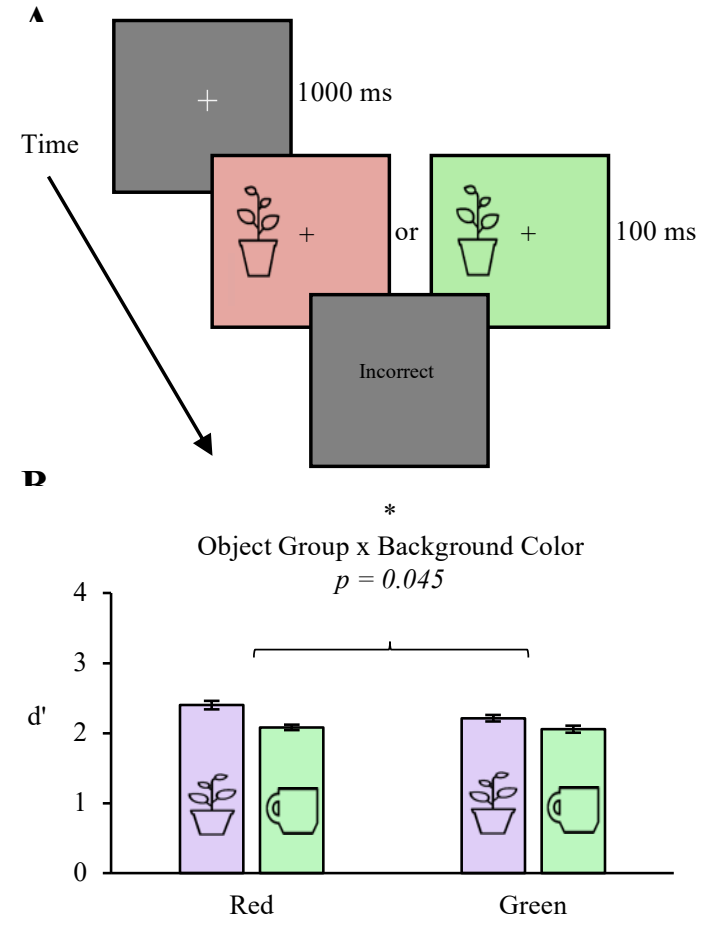

Figure 5. The paradigm and results of experiment 5. (A) Participants maintained fixation on the center cross. An object then appeared to the left or right of fixation. As in Experiment 1, participants were asked to detect the presence of small gap in the bottom outline of the object. The background color (red or green) was manipulated. (B) There was a significant main effect of object group $(F(1,25)=$ 17.171, $\left.\mathrm{p}<0.001, \eta_{\mathrm{p}}{ }^{2}=0.407\right)$ with non-manipulable objects having a higher average than manipulable objects $\left(\mathrm{M}_{\text {non-manipulable }}=2.25, \mathrm{M}_{\text {manipulable }}=2.02\right)$. There was no significant main effect of background color $(F(1,25)=$ $\left.2.138, \mathrm{p}=0.156, \eta_{\mathrm{p}}{ }^{2}=0.079\right)$ but there was a significant two-way interaction between object group and background color $\left(F(1,25)=4.444, \mathrm{p}=0.045, \eta_{\mathrm{p}}^{2}=0.151\right.$. 
We hypothesized that manipulable and non-manipulable objects, due to differential

298 recruitment of the two visual pathways, would elicit perceptual differences stemming from the

299 characteristic spatial and temporal resolutions associated with the magno- and parvo-cellular

300 inputs. In five experiments we provide strong evidence in support of behavioral consequences

301 driven by physiological and anatomical differences of the two visual pathways, and argue that

302 semantic knowledge of object utility guides processing along a particular pathway. If an object

303 has a strong action association, its processing will be largely determined by activity in the dorsal

304 pathway which is not found with objects that lack strong action association (Chao \& Martin,

305 2000; Noppeney et al., 2006; Mahon et al., 2007). The increased level of activity in the parietal

306 regions endows the perception of action-associated objects with greater access to the information

307 encoded in the magnocellular channel which preferentially courses through the dorsal pathway.

308 Without the enhanced dorsal pathway activity evoked by action associations, object

309 representation is more dependent on the ventral pathway, which has a higher ratio of

310 parvocellular channel information than does the dorsal pathway (Maunsell et al., 1990; Baizer et

311 al., 1991; Ferrera et al., 1992). Based on differential magno- and parvo-cellular input to the two

312 pathways, we predicted that the perception of objects with strong action associations results in an

313 increased temporal resolution and that the perception of objects without such associations results

314 in an increased spatial resolution. In the experiments reported here, we offer evidence that

315 objects are perceived with different spatial and temporal resolutions depending on object

316 semantic knowledge (namely, whether the object is manipulable or non-manipulable). In two

317 follow-up control experiments, further evidence was provided in support of the hypothesis that

318 the difference between manipulable and non-manipulable objects in the spatial task was driven 
319 by the semantic knowledge of the objects rather than possible low-level visual features. Namely, 320 it was observed that the effect is curtailed by impeding the access of object semantic knowledge

321 through inversion and it was observed that the perceptual differences between manipulable and

322 non-manipulable objects replicated with a separate set of more realistic object images (with a

323 different set of low-level object properties). Lastly, to test if the differing relative proportions of

324 magno- and parvo-cellular input are responsible for the perceptual differences that were observed

325 in our first four experiments, ambient red light was used to suppress activity of the magnocellular

326 channel. It was observed that this increased the perceptual difference in the spatial task between

327 manipulable and non-manipulable objects.

328 Results are strengthened by a set of various low-level controls imposed on the stimulus

329 set. Manipulable and non-manipulable object groups were controlled for size, hue, saturation,

330 visual clutter, and image value. Additionally, object groups were controlled for the degree of

331 elongation difference (i.e., aspect ratio) as this factor has been shown to drive some amount of

332 dorsal visual pathway selective for tools, which are a subset of manipulable object (Chen et al., 333 2018).

334 Based on the evidence provided, we argue that semantic knowledge of the manipulability

335 of objects, as defined by strong associations with an action appropriate for the item, generates the

336 perceptual differences between manipulable and non-manipulable objects. Previous studies have

337 shown that manipulable objects evoke a larger degree of dorsal pathway processing than do non-

338 manipulable objects (Chao \& Martin, 2000; Almeida et al., 2013). However, the origin of this

339 processing difference and consequent perceptual differences between manipulable and non-

340 manipulable objects is poorly understood. 
One possibility by which objects imbue semantic content related to manipulation is

342 implied manipulability. A potentially provocative investigation suggested that hand proximity (a

343 proxy for manipulability), leads to differences similar to the perceptual differences between

344 manipulable and non-manipulable objects (Gozli et al., 2012). Implied manipulability was

345 induced by varying the positions of participants' hands such that hands near the display implied

346 manipulability, while hands far from the display implied low manipulability. Importantly,

347 participants were presented with a simple circle shape, rather than manipulable or non-

348 manipulable objects. It was observed that when the participants' hands were positioned near the

349 experimental stimuli, participants' sensitivity to temporal blinks was heightened and when

350 participants' hands were far from the stimuli sensitivity to spatial gaps was heightened. In effect,

351 the placement of the participants' hand in this paradigm shifted the perception of the stimuli to

352 resemble either the non-manipulable objects, if the hands were far from the stimuli, or the

353 manipulable objects, if the hands were near the stimuli. It may be that when an object is learned

354 to be a manipulable, the neural representation of that object becomes associated with the near

355 hand effect reported by Gozli et al., (2012).

356 A possible mechanism linking the near-hand effect reported by Gozli et al. (2012) and the

357 perceptual difference between manipulable and non-manipulable objects derive from the bimodal

358 cells in the anterior parietal cortex (Graziano \& Gross, 1993). The bimodal cells have a

359 somatosensory receptive field covering a part of the hand and they have a visual receptive field

360 corresponding to a part of the visual field near their associated hand area. When an object is

361 shown, if that object does not have a strong action association, then the activity of the bimodal

362 cells is unaffected. After the organism has learned to use the object with their hands, the bimodal

363 cells will respond solely to the visual presentation of the object even in the absence of 
364 somatosensory input (Zhou \& Fuster, 2000). During object training, it has been shown that the

365 anterior parietal regions which contain the bimodal cells upregulate brain-derived neurotrophic

366 factor (BDNF), a protein which encourages the development of new synapses (Ishibashi et al.,

367 2002). The upregulation of BDNF may be what changes the circuit structure around the bimodal

368 cells to enable purely visual activation without the corresponding somatosensory input. Thus, the

369 bimodal cells and their ability to become solely visually activated may be a driver of the known

370 object selectivity of the dorsal processing pathway (Vaziri-Pashkam \& Xu, 2017; Kastner et al.,

371 2017; Freud et al., 2016) and lead to the dorsal pathway processing bias evoked by manipulable

372 objects (Chao \& Martin, 2000). The object-specific neural activity in the dorsal processing

373 pathway, divorced from its need for somatosensory input, and largely derived from

374 magnocellular input, could then be read out during an identification task with high temporal

375 resolution, leading to heightened temporal precision for manipulable objects.

376 Taken together, our results demonstrate that object semantic knowledge determines the

377 processing bias of the object (via dorsal or ventral bias) and evokes subsequent behavioral

378 repercussions for perception and for action. Our work underscores the need for careful

379 consideration of object semantic knowledge and its subsequent possible bias to either dorsal or

380 ventral pathway processing when object images are used not only in psychological research, but

381 in applied settings such as display and product designs, environmental design, and in the design

382 of various cognitive assistants. 


\section{Methods}

386 Participants. All participants were recruited from The George Washington University

387 participant pool, gave informed consent according to the George Washington University’s

388 institutional review board (IRB), were naïve to the purpose of the experiment, and all reported

389 normal or corrected-to-normal vision. In experiment 4, which utilized color stimuli no participant

390 reported colorblindness.

391 For experiment 1, 26 undergraduate students (19 female; average age: 19.2; 6 left-

392 handed) were recruited. No participant was removed from the analyses. For experiment 2, 27

393 undergraduate students were recruited. Twenty-six participants' data (14 female; average age:

394 19.0; 1 left-handed) were analyzed after one participant was cut for below chance accuracy in 395 one of the conditions. For experiment 3, 33 undergraduate students were recruited. Thirty

396 participants' data (26 female; average age: 19.07, 3 left-handed) were analyzed after eight

397 participants were cut for below chance accuracy in at least one of the conditions. For experiment

398 4, 34 undergraduate students were recruited. Twenty-five participants' data (14 female; average

399 age: 19.0; 1 left-handed) were analyzed after six participants were cut for not finishing the 400 experiment and nine participants were cut for having less than chance accuracy in at least one of

401 the conditions. For experiment 5, 26 undergraduate students were recruited. Twenty-five 402 participants' data (18 female; average age: 19.5, 0 left-handed) were analyzed after one 403 participant was cut for chance accuracy in all conditions.

405 Apparatus and Stimuli. All experiments were presented on a 24” Acer GN246 HL monitor 406 with a refresh rate of $144 \mathrm{~Hz}$, positioned at a distance of $60 \mathrm{~cm}$ from the viewer in a dark room. 407 The experiment was generated and presented using PsychoPy v1.82. 

objects drawn both from The Noun Project, an online repository of object icons and clip art

410 (https://thenounproject.com). The object stimuli consisted of 10 line-drawings of manipulable 411 objects, and 10 line-drawings of non-manipulable objects. The manipulable objects were as 412 follows: snow shovel, handsaw, plunger, screwdriver, hammer, wrench, knife, bottle opener, 413 spatula, and mug. The non-manipulable objects were: fire hydrant, picture frame, window, toilet, 414 candle, garbage can, water fountain, potted plant, fan, and lamp. The stimuli were displayed as 415 large as possible in a $4^{\circ} \times 4^{\circ}$ area and all stimuli were presented in black $(\mathrm{HSV}=0,0,0)$ on a dark gray background $(\mathrm{HSV}=0,0,50)$. All objects are displayed in Figure 1.

418 and aspect ratio (i.e., measure of elongation) for each object. There was no mean luminance 419 difference between object groups, an independent samples t-test $(t(18)=-0.960, p=0.350)$. There 420 was no significant difference in mean size (t-test $(t(18)=1.043, p=0.311)$, or aspect ratio $(t(18)$ $421=1.209, p=0.242$ ). The width of the bottom line was controlled for each object on which the gap 422 appeared. There was no significant difference between manipulable and non-manipulable objects 423 in bottom line width in an independent samples t-test $(t(18)=0.922, p=0.369)$.

424 For Experiment 3, the stimuli consisted of images of real-world, everyday objects drawn 425 from Google image search and manipulated in GIMP 2.10.4. The object stimuli consisted of 10 426 images of the same manipulable objects, and 10 images of the same non-manipulable objects used 427 in Experiments 1,2, 4, and 5. The stimuli were displayed as large as possible in a $4^{\circ} \times 4^{\circ}$ area and 428 all stimuli were presented in color on a dark gray background ( $\mathrm{HSV}=0,0,50)$. All objects are 429 displayed in Figure 1. 
Additionally, colored objects used in Experiment 3 were similarly controlled for low-level

431 differences by calculating the mean size, hue, saturation, value, aspect ratio, and visual clutter. In

432 analyzing mean size, there was no significant difference between manipulable and non-

433 manipulable objects in an independent samples t-test $(t(18)=-1.027, p=0.318)$. Additionally,

434 there were no significant differences for hue $(t(18)=-0.309, p=0.761)$, saturation $(t(18)=-0.031$,

$435 p=0.976)$, value $(t(18)=0.032, p=0.975)$, aspect ratio $(\mathrm{t}(18)=-0.094, p=0.927)$, or visual

436 clutter $(t(18)=0.616, p=0.545)$. Additionally, we controlled the width of the bottom line of each

437 object on which the gap would appear. There was no significant difference between manipulable

438 and non-manipulable objects in bottom line width in an independent samples t-test $(t(18)=0.557$, $439 p=0.584)$.

441 Design and Procedure. In Experiment 1, each trial began with a single central fixation point, 442 which subtended a visual angle of $1^{\circ} \times 1^{\circ}$. The central fixation point was rendered in white (HSV $443=0,0,100)$. After $1000 \mathrm{~ms}$ of fixation presentation, a single object line drawing would appear $4^{\circ}$ 444 to the left or right of the fixation point. The side of presentation was counterbalanced such that 445 each participant saw an equal number of stimuli on the left and on the right. The stimuli were 446 displayed as large as possible within a $4^{\circ} \times 4^{\circ}$ area and all stimuli were presented in color on a 447 dark gray background (HSV $=0,0,50)$. All objects are displayed in Figure 1.

448 In one half of the experiment, the object appeared with or without a spatial gap in the center 449 of the bottom line of the object. The objects had an equal probability of having a spatial gap or not 450 having a spatial gap. The objects appeared for $100 \mathrm{~ms}$ and participants were to report the presence 451 of the spatial gap by pressing the right control button (present) or the left control button (absent) 452 on the keyboard. Feedback was presented on incorrect trials only. 
A staircase procedure was used to calibrate the size of the gap to each object to ensure that 454 the gap was equally perceptible across each object regardless of each objects' individual 455 characteristics (Pelli and Bex, 2013). Sixteen undergraduate students (13 female; average age: 18.8; 4564 left-handed) from the George Washington University participated in exchange for course credit. 457 In each trial the object would be presented with or without the spatial gap which would begin with 458 a size of $0.025^{\circ}$ visual angle. If the gap was detected correctly for two consecutive trials, the gap 459 decreased by one $0.005^{\circ}$ step. If the gap was missed, or a false alarm was made to the absence of 460 a gap, for two consecutive trials, the gap was increased by one $0.005^{\circ}$ step. The gap was calibrated 461 until twenty trials had been completed or until the staircase reversed direction (2 correct followed 462 by 2 incorrect or vice versa) three times. The gap size for each object is displayed in figure 1.

463 In the other half of the experiment, the object appeared with or without a temporal blink. 464 The objects had an equal probability of having a temporal blink or not having a temporal blink. 465 The blinks were $16 \mathrm{~ms}$ long. The object first appeared for $96 \mathrm{~ms}$, followed by the temporal blink, 466 and the object then appeared a second time for $32 \mathrm{~ms}$. Participants were to report the presence of 467 the temporal blink by pressing the right control button and absence of the temporal blink by 468 pressing the left control button on the keyboard. Feedback was presented on incorrect trials only. 469 The presentation of stimulus type was counterbalanced across participants such that half of 470 the participants were first presented with spatial gaps and half of the participants were presented 471 with temporal blinks first. Participants completed a total of 720 trials broken into 6 blocks, 360 472 trials of spatial gaps and 360 trials of temporal blinks.

473 Experiment 2 was identical to Experiment 1 except each stimulus was presented upside 474 down. The spatial gap was presented in the same place as it was in experiment 1 relative to the 475 object. 
Experiment 3 was identical to experiment 1, except more realistic images were used instead

477 of line drawings. Similarly to the line drawing stimuli, a staircase procedure was used to calibrate

478 the size of the gap to each object to ensure that the gap would be equally perceptible across each

479 object regardless of each objects' individual characteristics (Pelli and Bex, 2013). Twenty three

480 undergraduate students (18 female; average age: 18.9; 4 left-handed) from the George Washington

481 University participated exchange for course credit. In each trial the object would be presented with

482 or without the spatial gap which would begin with a size of $0.025^{\circ}$ visual angle. If the gap was

483 detected correctly for two consecutive trials, the gap would be made smaller by one $0.005^{\circ}$ step.

484 If the gap was not detected, or a false alarm was made to the absence of a gap, for two consecutive

485 trials, then the gap would be made larger by one $0.005^{\circ}$ step. Calibration was done until twenty

486 trials had been completed or until the staircase reversed direction ( 2 correct followed by 2 incorrect

487 or vice versa) three times. The gap size for each object is displayed in figure 1.

488 Experiment 4 was similar in trial structure to the temporal blink condition of Experiment

489 1, except instead of temporal blink detection each trial had a temporal blink that was either short

490 or long in duration. The objects had an equal probability of having a short or a long temporal

491 blink. In the short blink condition, the object first appeared for $96 \mathrm{~ms}$, followed by a $16 \mathrm{~ms}$

492 temporal blink, and then the object appeared a second time for $32 \mathrm{~ms}$. In the long blink

493 condition, the object first appeared for $64 \mathrm{~ms}$, followed by a $48 \mathrm{~ms}$ temporal blink, and then the

494 object was presented for a second time for $32 \mathrm{~ms}$. Participants were to report a presence of a

495 short temporal blink by pressing the 'c' key and a long temporal blink by pressing the ' $\mathrm{m}$ ' key on

496 the keyboard. Feedback was presented exclusively on incorrect trials. Participants completed a

497 total of 720 trials broken into 6 blocks, 360 trials of short blink duration and 360 trials of long

498 blink duration. 
Experiment 5, was identical to the spatial gap procedure of Experiment 1 except the 500 background color was either green $(\mathrm{HSV}=110,30,90)$ or red $(\mathrm{HSV}=5,30,90)$. The background 501 color was counterbalanced across participants such that half of the participants would first be 502 presented with the green background and half of the participants would first be presented with the 503 red background. Participants completed a total of 720 trials broken into 6 blocks, 360 trials of 504 green backgrounds and 360 trials of red backgrounds.

505

506 Data Analyses. Data for each experiment were analyzed using repeated-measures analysis of 507 variance with an $\alpha$ set to 0.05 . Two-sample t-test were also employed where indicated in the text 508 and were two-tailed. Anticipatory response times faster than $250 \mathrm{~ms}$ and any response times 509 longer than $2000 \mathrm{~ms}$ were removed from analysis $(2.67,2.4,5.59,12.1$, and $2.95 \%$ of the data 510 was removed from experiments 1, 2, 3, 4, and 5 respectively). Mean response times for each 511 condition in each experiment were calculated for each subject. For each analysis, all 512 assumptions, including normality and equality of variances, were formally tested and confirmed. 
514 Code availability. The custom Python programs generated for this study are available from the 515 corresponding authors on reasonable request.

516 Data availability. The data that support the findings of this study are available from the 517 corresponding author upon reasonable request. 


\section{References}

520 Almeida, J., Mahon, B. Z., Nakayama, K., \& Caramazza, A. (2008). Unconscious processing 521 dissociates along categorical lines. Proceedings of the National Academy of Sciences of the $522 \quad$ United States of America, 105(39), 15214-15218. https://doi.org/10.1073/pnas.0805867105

523 Almeida, J., Mahon, B. Z., \& Caramazza, A. (2010). The role of the dorsal visual processing 524 stream in tool identification. Psychological Science: A Journal of the American 525 Psychological Society / APS, 21(6), 772-778. https://doi.org/10.1177/0956797610371343

Almeida, J., Fintzi, A. R., \& Mahon, B. Z. (2013). Tool manipulation knowledge is retrieved by way of the ventral visual object processing pathway. Cortex, 49(9), 2334-2344. https://doi.org/10.1016/j.cortex.2013.05.004

Baizer, J. S., Ungerleider, L. G., \& Desimone, R. (1991). Organization of visual inputs to the inferior temporal and posterior parietal cortex in macaques. Journal of Neuroscience, 11(1),

Blasdel, G. G., \& Lund, J. S. (1983). Termination of Afferent Axons in Macaque Striate Cortex. Journal of Neuroscience, 3(7), 1389-1413.

534 Chao, L. L., \& Martin, A. (2000). Representation of manipulable man-made objects in the dorsal stream. NeuroImage, 12(4), 478-484. https://doi.org/10.1006/nimg.2000.0635

536 Chen, J., Snow, J. C., Culham, J. C., \& Goodale, M. A. (2018). What Role Does "Elongation" 537 Play in "Tool-Specific" Activation and Connectivity in the Dorsal and Ventral Visual 538 Streams? Cerebral Cortex (New York, N.Y., 1991), 28(4), 1117-1131. https://doi.org/10.1093/cercor/bhx017

540 Collaboration, O. S. (2015). Estimating the reproducibility of psychological science. Science, $541 \quad 349(6251)$. https://doi.org/10.1126/science.aac4716 
542 Derrington, A. M., \& Lennie, P. (1984). Spatial and temporal contrast sensitivities of neurons in 543 lateral geniculate nucleus of macaque. The Journal of Physiology, 357(1), 219-240.

$544 \quad$ https://doi.org/10.1113/jphysiol.1984.sp015498

545 DeYoe, E. A., \& van Essen, D. C. (1988). Concurrent processing streams in monkey visual 546 cortex. Trends in Neurosciences, 11(5), 219-226. https://doi.org/10.1016/0166$547 \quad 2236(88) 90130-0$

548 Diamond, R., \& Carey, S. (1986). Why Faces Are and Are Not Special. An Effect of Expertise. $549 \quad$ Journal of Experimental Psychology: General, 115(2), 107-117. $550 \quad$ https://doi.org/10.1037/0096-3445.115.2.107

551 Fang, F., \& He, S. (2005). Cortical responses to invisible objects in the human dorsal and ventral 552 pathways. Nature Neuroscience, 8(10), 1380-1385. https://doi.org/10.1038/nn1537

553 Ferrera, V. P., Nealey, T. A., \& Maunsell, J. H. R. (1992). Mixed parvocellular and 554 magnocellular geniculate signals in visual area V4. Nature, 358(6389), 756-758. https://doi.org/10.1038/358756a0

556 Freud, E., Plaut, D. C., \& Behrmann, M. (2016). 'What' Is Happening in the Dorsal Visual 557 Pathway. Trends in Cognitive Sciences, 20(10), 773-784.

$558 \quad$ https://doi.org/10.1016/j.tics.2016.08.003

559 Gozli, D. G., West, G. L., \& Pratt, J. (2012). Hand position alters vision by biasing processing 560 through different visual pathways. Cognition, 124(2), 244-250.

$561 \quad$ https://doi.org/10.1016/j.cognition.2012.04.008

562 Graziano, M. S. A., \& Gross, C. G. (1993). A bimodal map of space: somatosensory receptive 563 fields in the macaque putamen with corresponding visual receptive fields. Experimental 564 Brain Research, 97(1), 96-109. https://doi.org/10.1007/BF00228820 
Ishibashi, H., Hihara, S., Takahashi, M., Heike, T., Yokota, T., \& Iriki, A. (2002). Tool-use learning selectively induces expression of brain-derived neurotrophic factor, its receptor trkB, and neurotrophin 3 in the intraparietal multisensory cortex of monkeys. Cognitive Brain Research, 14(1), 3-9. https://doi.org/10.1016/S0926-6410(02)00056-3

James, T. W., Culham, J., Humphrey, G. K., Milner, A. D., \& Goodale, M. A. (2003). Ventral occipital lesions impair object recognition but not object-directed grasping: An fMRI study. Brain, 126(11), 2463-2475. https://doi.org/10.1093/brain/awg248

Karnath, H. O., Rüter, J., Mandler, A., \& Himmelbach, M. (2009). The anatomy of object recognition - Visual form agnosia caused by medial occipitotemporal stroke. Journal of Neuroscience, 29(18), 5854-5862. https://doi.org/10.1523/JNEUROSCI.5192-08.2009

Kastner, S., Chen, Q., Jeong, S. K., \& Mruczek, R. E. B. (2017). A brief comparative review of primate posterior parietal cortex: a novel hypothesis on the human toolmaker. Neuropsychologia, 105, 123-134. https://doi.org/10.1016/j.neuropsychologia.2017.01.034.A

Kravitz, D. J., Saleem, K. S., Baker, C. I., \& Mishkin, M. (2011). A new neural framework for visuospatial processing. Nature Reviews Neuroscience, 12(4), 217-230. https://doi.org/10.1038/nrn3008

Kravitz, D. J., Saleem, K. S., Baker, C. I., Ungerleider, L. G., \& Mishkin, M. (2013). The ventral visual pathway: An expanded neural framework for the processing of object quality. Trends in Cognitive Sciences, 17(1), 26-49. https://doi.org/10.1016/j.tics.2012.10.011

Leonova, A., Pokorny, J., \& Smith, V. C. (2003). Spatial frequency processing in inferred PCand MC-pathways. Vision Research, 43(20), 2133-2139. https://doi.org/10.1016/S00426989(03)00333-X 
Leventhal, A. G., Rodieck, R. W., \& Dreher, B. (1981). Retinal ganglion cell classes in the old world monkey: Morphology and central projections. Science, 213(4512), 1142-1144. https://doi.org/10.1126/science.7268423

591 Livingstone, M. S., \& Hubel, D. H. (1988). Do the relative mapping densities of the magno- and 592 parvocellular systems vary with eccentricity? Journal of Neuroscience, 8(11), 4334-4339. $593 \quad$ https://doi.org/10.1523/jneurosci.08-11-04334.1988

594 Mahon, B. Z., Milleville, S. C., Negri, G. A. L., Rumiati, R. I., Caramazza, A., \& Martin, A. 595 (2007). Action-Related Properties Shape Object Representations in the Ventral Stream. Neuron, 55(3), 507-520. https://doi.org/10.1016/j.neuron.2007.07.011

597 Maunsell, J. H. R., Ghose, G. M., Assad, J. A., McAdams, C. J., Boudreau, C. E., \& Noerager, 598 B. D. (1999a). Visual response latencies of magnocellular and parvocellular LGN neurons 599 in macaque monkeys. Visual Neuroscience, 16(1), 1-14.

$600 \quad$ https://doi.org/10.1017/S0952523899156177

601 Maunsell, J. H. R., Ghose, G. M., Assad, J. A., McAdams, C. J., Boudreau, C. E., \& Noerager, 602 B. D. (1999b). Visual response latencies of magnocellular and parvocellular LGN neurons 603 in macaque monkeys. Visual Neuroscience, 16(1), 1-14.

$604 \quad$ https://doi.org/10.1017/S0952523899156177

605 Maunsell, J. H. R., Nealey, T. A., \& DePriest, D. D. (1990). Magnocellular and parvocellular 606 contributions to responses in the middle temporal visual area (MT) of the macaque monkey. 607 Journal of Neuroscience, 10(10), 3323-3334. https://doi.org/10.1523/jneurosci.10-10608 03323.1990 
McAnany, J. J., \& Alexander, K. R. (2008). Spatial frequencies used in Landolt C orientation judgments: Relation to inferred magnocellular and parvocellular pathways. Vision Research, 48(26), 2615-2624. https://doi.org/10.1016/j.visres.2008.02.012

Mishkin, M., \& Ungerleider, L. G. (1982). Contribution of striate inputs to the visuospatial functions of parieto-preoccipital cortex in monkeys. Behavioural Brain Research, 6(1), 5777. https://doi.org/10.1016/0166-4328(82)90081-X

Nassi, J. J., \& Callaway, E. M. (2009). Parallel processing strategies of the primate visual system. Nature Reviews Neuroscience, 10(5), 360-372. https://doi.org/10.1038/nrn2619

Noppeney, U., Price, C. J., Penny, W. D., \& Friston, K. J. (2006a). Two distinct neural mechanisms for category-selective responses. Cerebral Cortex, 16(3), 437-445. https://doi.org/10.1093/cercor/bhi123

Noppeney, U., Price, C. J., Penny, W. D., \& Friston, K. J. (2006b). Two distinct neural mechanisms for category-selective responses. Cerebral Cortex, 16(3), 437-445. https://doi.org/10.1093/cercor/bhi123

Perry, V. H., Oehler, R., \& Cowey, A. (1984). Retinal ganglion cells that project to the dorsal lateral geniculate nucleus in the macaque monkey. Neuroscience, 12(4), 1101-1123. https://doi.org/10.1016/0306-4522(84)90006-X

Pokorny, J., \& Smith, V. C. (1997). Psychophysical signatures associated with magnocellular and parvocellular pathway contrast gain: comment. Journal of the Optical Society of America A, 14(9), 2477-2486. https://doi.org/10.1364/josaa.15.002440

Vaziri-Pashkam, M., \& Xu, Y. (2017). Goal-directed visual processing differentially impacts human ventral and dorsal visual representations. Journal of Neuroscience, 37(36), 87678782. https://doi.org/10.1523/JNEUROSCI.3392-16.2017 
632 Wiesel, T. N., \& Hubel, D. H. (1966). Spatial and chromatic interactions in the lateral geniculate 633 body of the rhesus monkey. Journal of Neurophysiology, 29(6), 1115-1156.

634 https://doi.org/10.1152/jn.1966.29.6.1115

635 Zhou, Y. di, \& Fuster, J. M. (2000). Visuo-tactile cross-modal associations in cortical

636 somatosensory cells. Proceedings of the National Academy of Sciences of the United States

637 of America, 97(17), 9777-9782. https://doi.org/10.1073/pnas.97.17.9777

638 
641 BCS-2022572 to S.S. The funders had no role in study design, data collection and analysis,

642 decision to publish, or preparation of the manuscript. We thank D. Kravitz and M. Behrmann for

643 insightful comments on an earlier version of this manuscript. Special thanks go to E. Awh for

644 suggesting a temporal manipulation adopted for Experiment 4.

645

646 
Figures

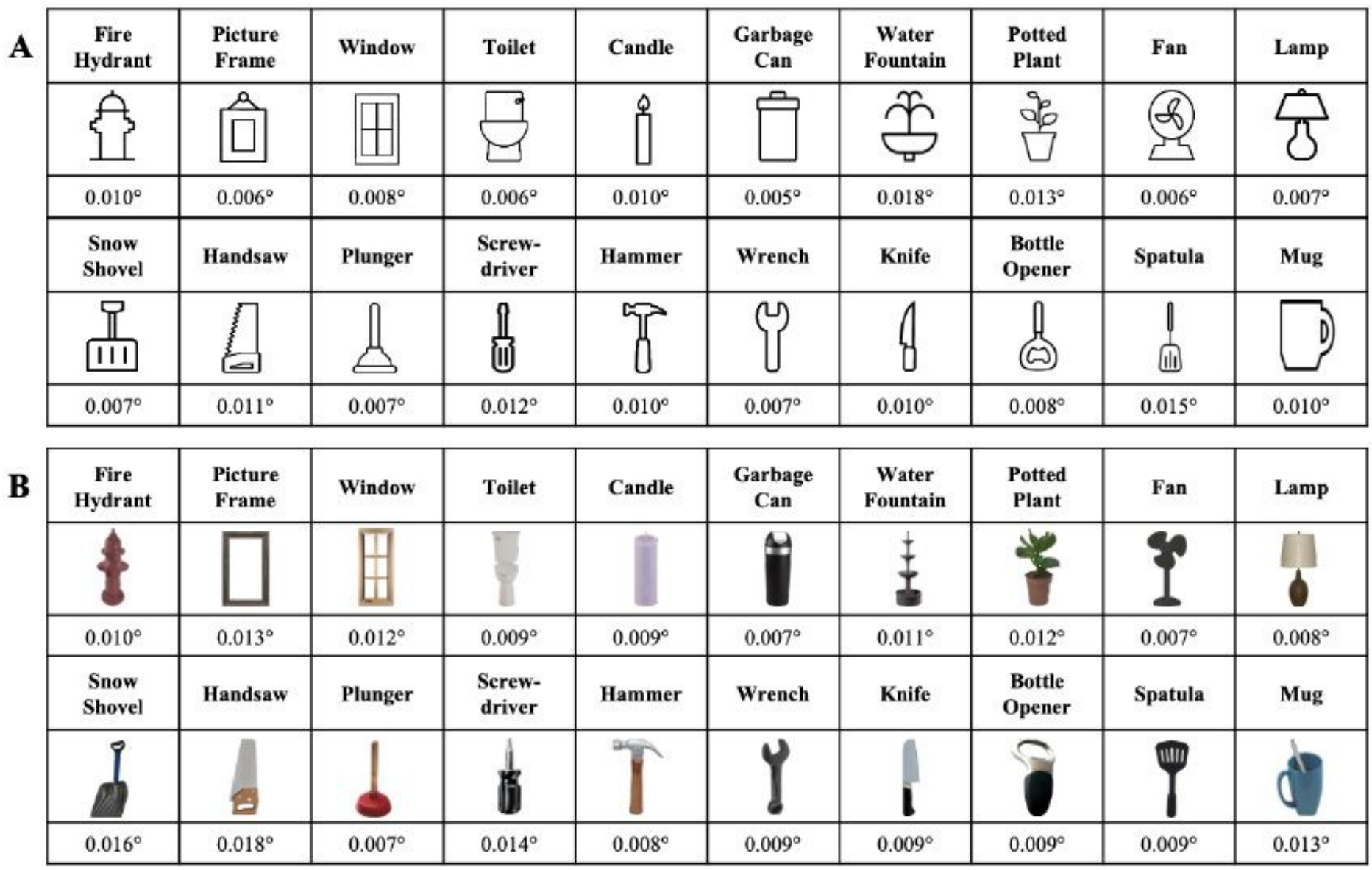

\section{Figure 1}

Stimulus set used in the experimental paradigms. (A) Line drawings of manipulable and non-manipulable objects used in Exp. 1. (B) Pictures of real-world manipulable and non-manipulable objects used in the second control manipulation for Exp. 1. The numbers beneath each set of objects correspond to the size of the spatial gap titrated to each object. 

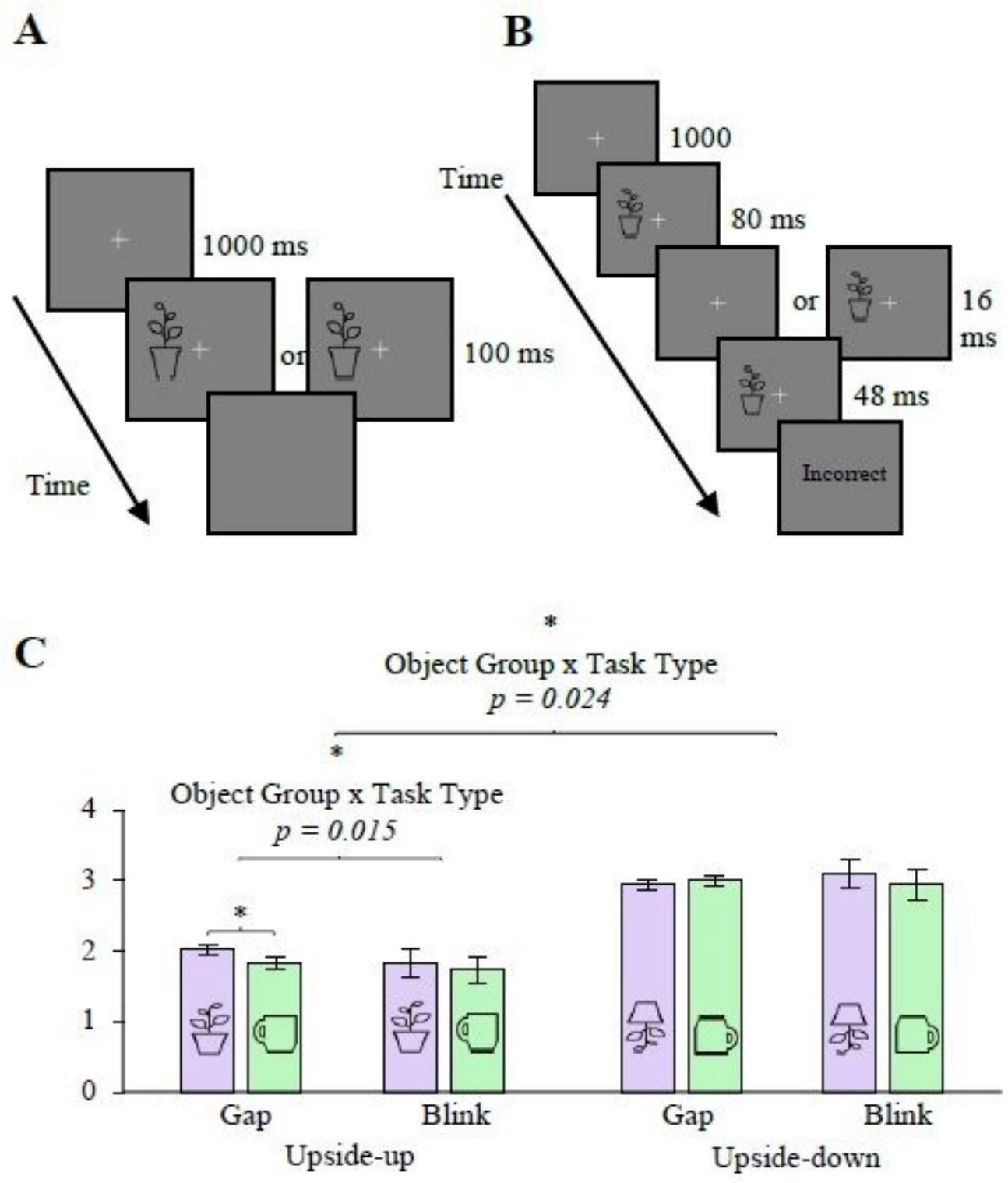

Figure 2

The paradigm and results for Experiments 1 and 2. Participants maintained fixation on the center cross. An object appeared to the left or right of fixation. (see Manuscript file for full figure legend) 


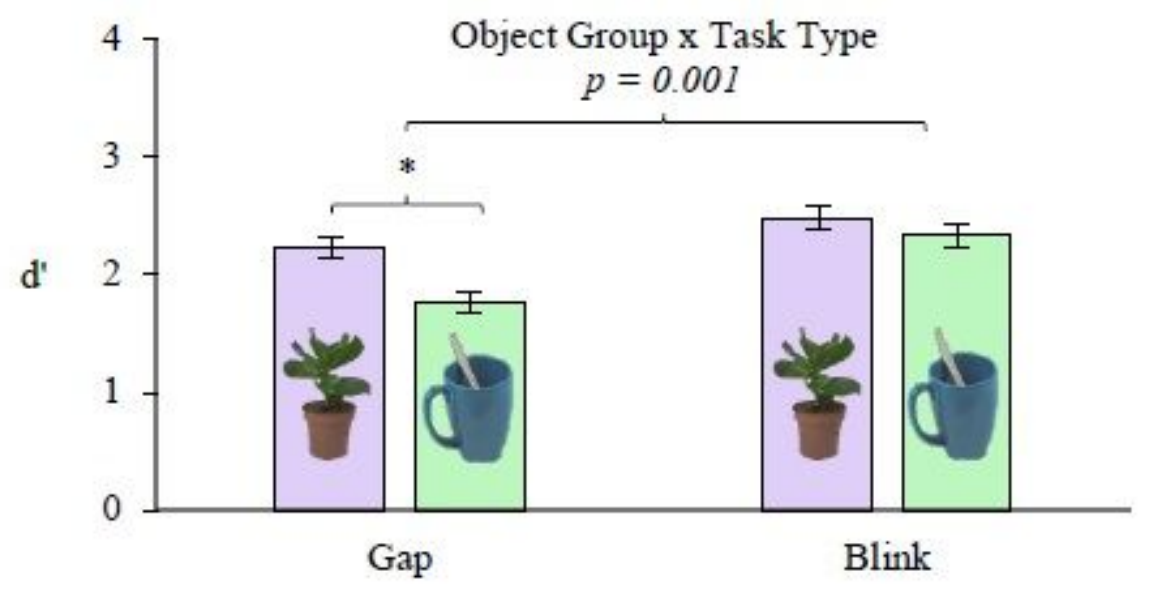

Figure 3

Results from the second control manipulation of experiment one in which real object images were used instead of line drawing images. (see Manuscript file for full figure legend) 

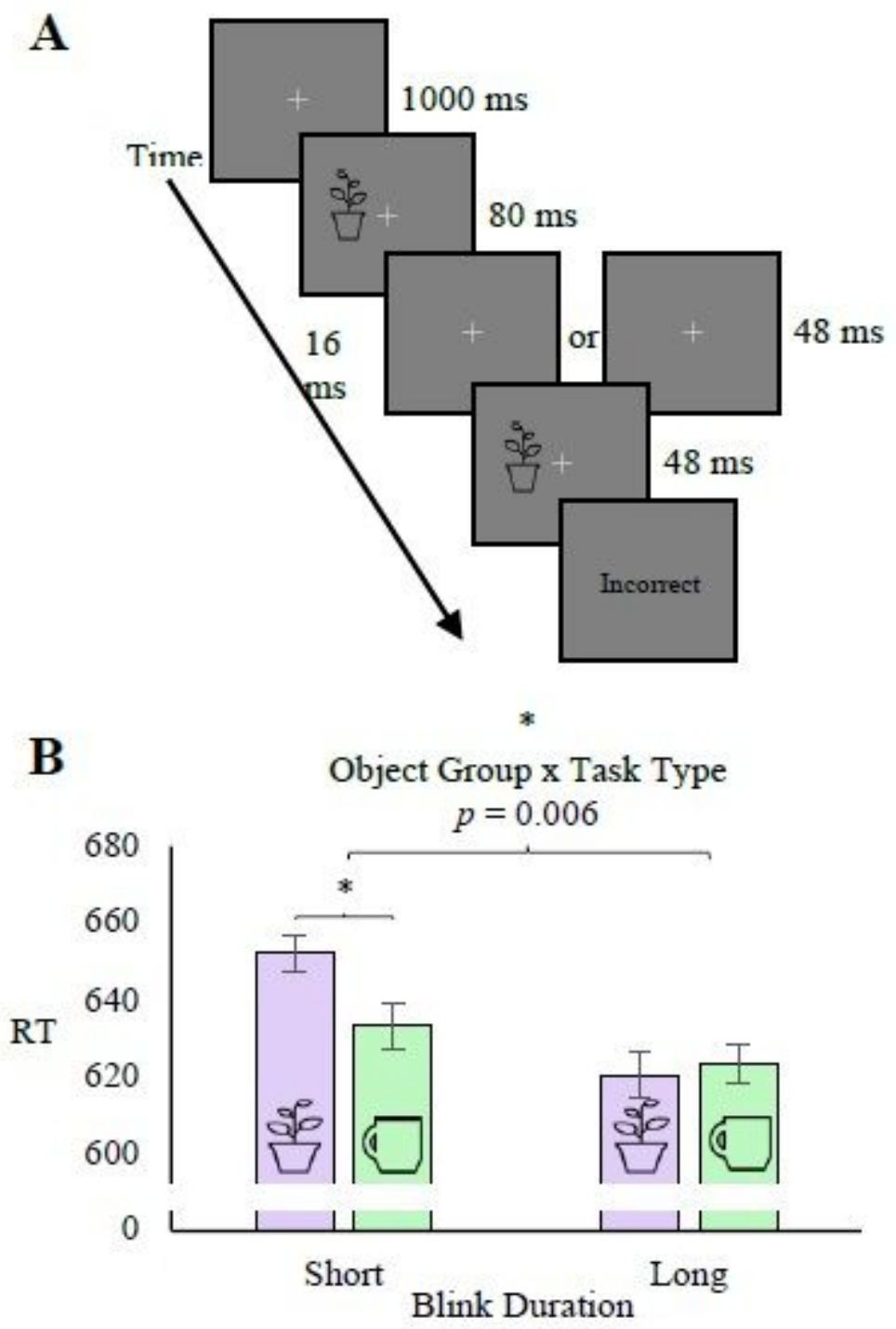

Figure 4

The paradigm and results for experiment 4. (see Manuscript file for full figure legend) 

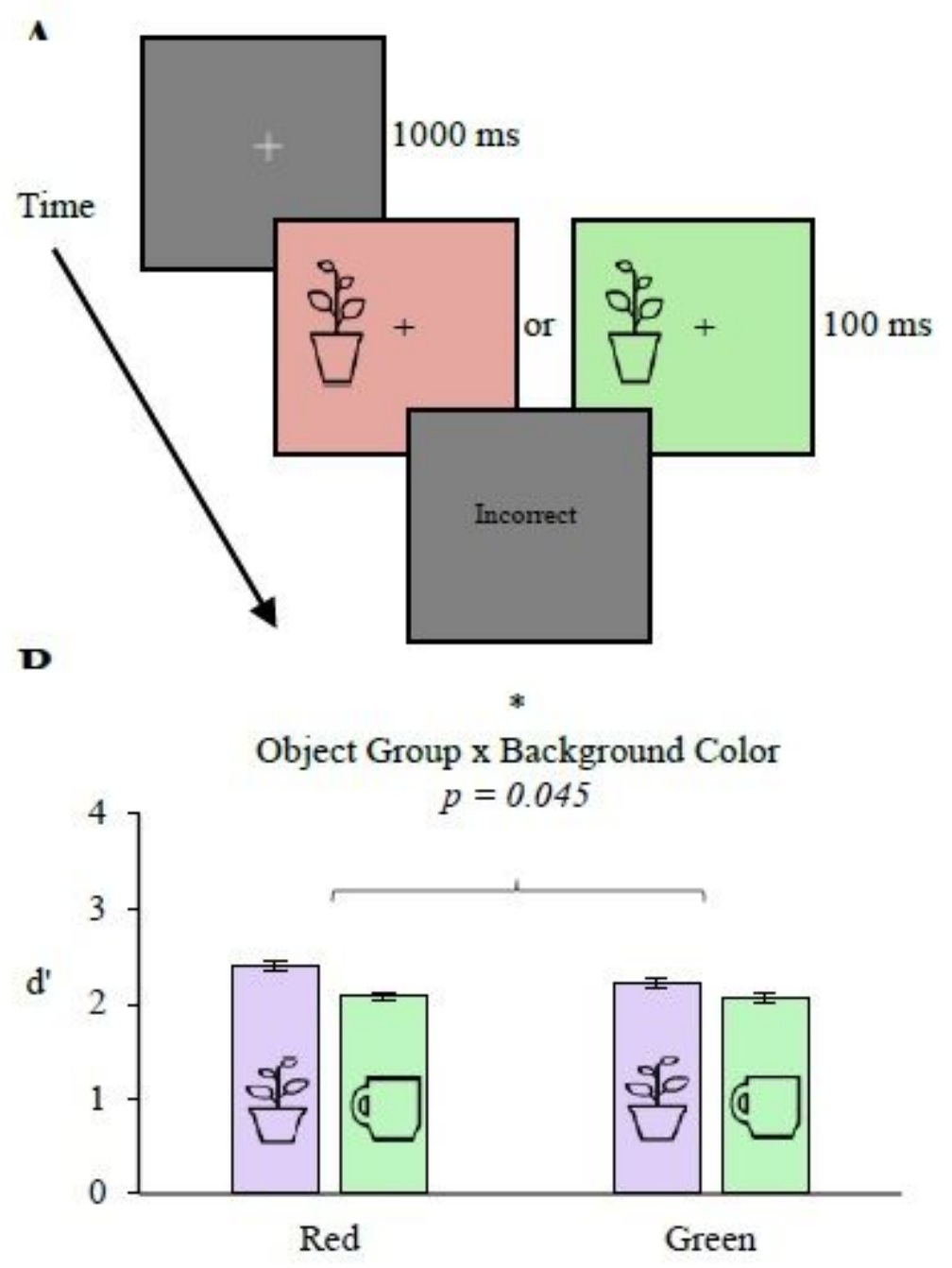

Figure 5

The paradigm and results of experiment 5. (see Manuscript file for full figure legend) 